\title{
NOTE ON DATES AND TRANSLITERATIONS
}

All dates are Common Era unless otherwise noted. In a few places, it is indicated that dates are given as A.H., which stands for "after the hijra," the beginning of the Islamic calendar.

Transliteration of Chinese follows the Hanyu Pinyin system. Persian and Arabic follow the International Journal of Middle Eastern Studies standard, without the diacritics. 
